\title{
A Blockchain-based Value Added Tax (VAT) System: Saudi Arabia as a Use-Case
}

\author{
Ahmad Alkhodre ${ }^{1}$ \\ Islamic University of Madinah
}

\author{
Salman $\mathrm{Jan}^{3}$ \\ Malaysian Institute of Information Technology \\ Universiti Kuala Lumpur \& University of Peshawar
}

\author{
Shah Khusro ${ }^{5}$ \\ University of Peshawar
}

\author{
Toqeer $\mathrm{Ali}^{2}$ \\ Islamic University of Madinah
}

\author{
Yazed Alsaawy \\ Islamic University of Madinah
}

\author{
Muhammad Yasar 6 \\ Malaysian Institute of Information Technology \\ Universiti Kuala Lumpur
}

\begin{abstract}
Businesses need trust to confidently perform trade among each other. Centralized business models are the only mature solutions available to perform trades over the Internet. However, they have many problems which includes but are not limited to the fact that these create bottleneck on the server as well as requires trusted third parties. Recently, decentralized solutions have gained significant popularity and acceptance for future businesses. The wide acceptance of such systems is indeed due to the trust management among various untrusted business stakeholders. Many solutions have been proposed in this regard to provide de-centralized infrastructure for various business models. A standard solution that is acceptable to the industry is still in demand. Hyperledger umbrella Blockchain projects, that are supported by IBM and many other industry big players are gaining popularity due to its efficient and pluggable design. In this study, the author present the idea of utilizing Blockchain to design a Value-Added Tax (VAT) system for Saudi Arabia's newly introduced tax system. The reason to select this business model for VAT is twofold. First, it provides an untampered distributed ledger, which cannot be deceived by any party. Each transaction in the system cannot go unnoticed by the smart contract. Secondly, it provides a transparent record, and updates all involved parties regarding each activity performed by stakeholders. The newly proposed system will provide a transparent database of VAT transactions according to our smart contract design and at each stage of supply chain, tax will be deducted and stored on peer-to-peer network via consensus process. The author believes that the proposed solution will have significant impact on VAT collection in the Kingdom of Saudi Arabia.
\end{abstract}

Keywords-VAT; hyperledger; blockchain; consensus; decentralized network

\section{INTRODUCTION}

Value Added Tax (VAT), is a self credit mechanism of indirect accumulative tax related to consumption of goods or services which is imposed on the end user as chain of business [1], [2]. VAT is collected from individual customers and is paid back to the Government. VAT collection is an important process of a state administrative authority that allows a government to generate revenue from active honest tax payers and if the tax payment is not made properly then the dishonest payers may reduce the tax liabilities to their own level of choices. Consequent to non compliance in tax payment and tax fraud, countries and governments receive losses in tax collection [3], [4]. It has also been observed that significant time and resources are consumed during the audit when the tax gap is identified.

VAT is implemented at the stage of production and distribution to increase the inland revenue. The noncompliance of VAT implementation results in the VAT gap [5] that represents the difference between estimated tax and actual tax collected. There are a number of reasons for the VAT gap which may include envision, fraud, bankruptcies, and insolvencies relating finance, etc [6].

In Saudi Arabia, VAT is introduced as a standard rate of 5\% with effect from January 2018 (Rabi Al-Thani 14, 1439) with exception of some goods and services. The sectors for which VAT is avoided includes health, education and transportation [7]. However, VAT is implemented on services and things like healthcare and treatment [8], medicine and medical equipments, import and exports [9] clothes, fuel i.e. food, petrol, \& diesel, utility bills and hotel rooms etc. Failing to pay VAT, General Authority of Zakat \& Tax of Saudia Arabia may like to impose appropriate penalties on taxpayers for violating VAT rules as set forth by the Law or implementing regulations. However, there are difficulties due to the manual implementation of the tax collection procedure which cannot be neglected [10].

Generally tax invoices are proof of tax collection. A tax invoice is a proof that the seller has collected the VAT from the buyer. A VAT previously paid by a buyer of good is partly recovered from the next buyer of the same good and services. During the VAT reporting period, the difference is calculated among tax invoice paid and tax invoice received. Only the taxable person for VAT purposes can generate tax invoices [11].

\section{A. VAT Mechanism as Adopted by Saudi Arabia}

There are 135,906 VAT registered businesses in the Saudi Arabia. Upon purchase of taxable service or goods, buyer pays VAT to the seller along with price of the service or good. This information is reflected in the invoice issued by the seller. The buyer can then use this invoice to show he/she is an active tax payer and further to reduce her overall tax where required.

At the point when a VAT-enlisted business offers a decent or benefit, it charges - accepting a standard case - an additional 
$5 \%$ of VAT over the business cost. The business will apply that $5 \%$ to every single qualified sales independently from its income with the end goal to later dispatch a bit of it to the administrative authority or government. The VAT that a business gathers on its deals is called Output VAT. The VAT that a business pays back to its providers is called Input VAT. With the end goal to ascertain the amount they owe to GAZT, every business will take note of the amount of VAT it has gathered from clients and subtract from it the aggregate VAT it paid in a similar period. A detailed process of VAT collection in KSA is described in Fig. 1 while an example is also provided in Table I.

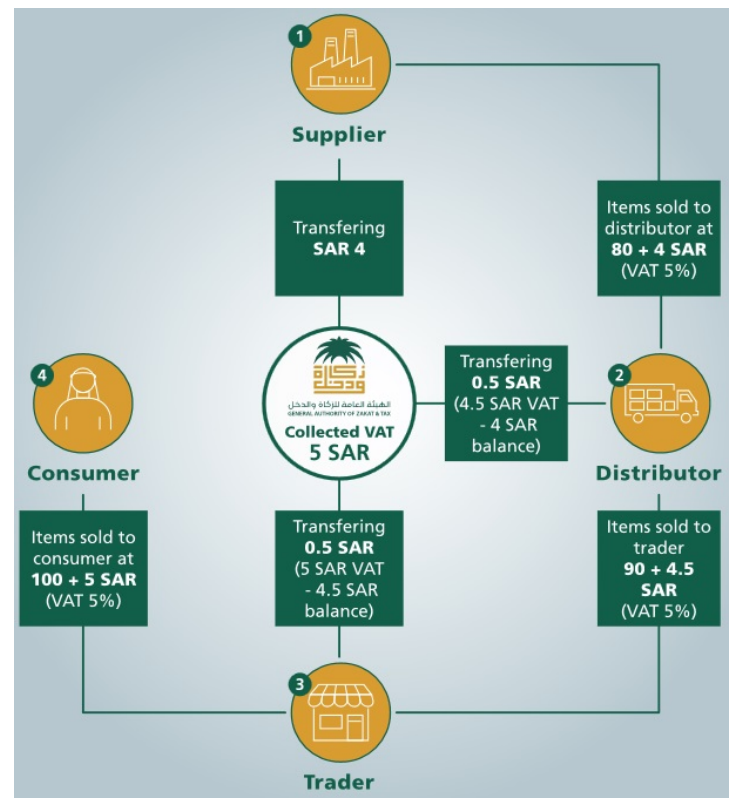

Fig. 1. VAT collection mechanism in KSA

\section{B. Centralized vs. Decentralized Businesses}

Famous examples of centralized business-to-business and customer-to-business e-commerce systems include ebay, Amazon [12], [13], etc. However, to bring trust into these systems, third party authorities are required. Due to these trusted entities customers make product selection, provide credit card information, make checkouts and payments so all tasks are indeed centralized and all the actions are initiated by users keeping in view trust components. Similar is the case with banks that are in fact centralized and trusted entities which are in turn authorized by some other entities till it reaches the Government bodies. In short, all types of businesses that includes businessto-business, business-to-customers, customer-to-business and customer-to-customer are indeed centralized systems and involve trust parties. So, a chain-of-trust is developed which originates in some place and ends in other that leads to no direct trust involved with clients [14]. Further, it includes the bottleneck on the central entity.

We have on the other hand decentralized systems like the Blockchain, that is in fact an evolution business oriented decentralized and heterogeneous solution involving a chain-oftrust among various peer entities. It has decentralized trust in a manner that all communication is allowed in this paradigm while there is no need of a third party, i.e. it ensures that communication among the parties or nodes of the Blockchain is trusted [15], [16], [17]. In fact, it stores all the communication among the two or more untrusted parties on an untampered ledger. This ledger is verified by many peer nodes before storing it. This simple mechanism gives trust to all parties involved in the business transaction that nobody can forge with the information.

Cryptocurrencies [18], for example, bitcoin [19], etherium [20] infrastructure that provides peer-to-peer business model. All are decentralized environments and involves trust among nodes without the need for a third party. It is also important to mention here that decentralized entities, such as, Blockchain has been tested with many models [21]. Various models have been presented to implement blockchain technologies. However, ought most priority need to be paid in opting a model for the businesses that is adopted and backed by various industry giants.

In [22] provides an implementation of Blockchain that has features of solving consensus and can manage and resolve disputes among various entities. However, it could not be adopted by large industrial players as those implementations were too specific. The author selected a model implementation of Blockchain that is adopted by the industry, the Hyperledger Fabric. Majority of banks, Intel and Google, etc. have support in these umbrella projects.

Value Added Tax (VAT) is recently imposed by the government of Saudi Arabia for the first time. However, there is a need for an automated system that can keep track of millions of transactions generated from a supply-chain in the country for VAT. A traditional solution is to design and implement a centralized system, however, due to aforementioned reasons it is necessary to adopt new technologies which have more flexibility. In this regard, Blockchain is quite feasible for VAT implementation because most of the future application are going towards it. All the stakeholders in the supplychain system will become part of Blockchain network and all transaction will go via the Blockchain system, setup by the government authorities. Smart-contract will be introduced to keep track of valid transaction and propagation on the Blockchain network.

The aim of conducting the research is to critically examine the existing VAT requirements and to provide implementation of model that can effectively collect VAT without the need of a third party and aligned with vision 2030 of Saudi Arabia while the objectives of studies are provided hereunder:

1) To Design Hyperledger Fabric and Composer infrastructure for supply chain management.

2) To design an end-to-end VAT implementation on a decentralized network.

3) To implement newly designed VAT system on Hyperledger composer and hyperledger fabric for implementation of VAT and setting up various peers and order nodes on Blockchain network.

Decentralized network and VAT are newly introduced in Saudi Arabia. For the implementation of the same authors adopted decentralized system. We opt Blockchain and within the Blockchain the author adopted a generalized system that 
(IJACSA) International Journal of Advanced Computer Science and Applications,

TABLE I. EXPLANATION OF VAT PROCESS THROUGH PURCHASE OF COMPUTERS

\begin{tabular}{|c|c|c|c|c|c|}
\hline $\begin{array}{l}\text { VAT } \\
\text { (transactions) }\end{array}$ & Price of Item & Tax per item & $\begin{array}{l}\text { Items bought } \\
\text { or sold }\end{array}$ & Total Tax & Name of Tax \\
\hline $\begin{array}{l}\text { Computers } \\
\text { bought from } \\
\text { Manufacturer }\end{array}$ & SAR 1,000 & 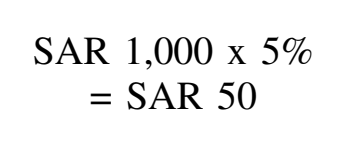 & 100 & $\begin{aligned} & \text { SAR } 50 \mathrm{x} \\
& 100 \mathrm{PCs} \\
= & \mathrm{SAR} 5,000\end{aligned}$ & Input-VAT \\
\hline $\begin{array}{l}\text { Computers } \\
\text { sold to customer }\end{array}$ & SAR 1,200 & $\begin{array}{c}\text { SAR } 1,200 \times 5 \%= \\
\text { SAR } 60\end{array}$ & 100 & $\begin{aligned} & \text { SAR } 60 x \\
& 100 \text { PCs } \\
= & \text { SAR } 6,000\end{aligned}$ & Output-VAT \\
\hline $\begin{array}{l}\text { VAT owed to } \\
\text { Govt }\end{array}$ & & $\begin{array}{l}\text { VAT due }= \\
\text { (SAR } 6,000-\end{array}$ & $\begin{array}{l}\text { tput VAT - Inp } \\
\text { AR } 5,000=S A\end{array}$ & $\begin{array}{l}\text { VAT } \\
1,000)\end{array}$ & \\
\hline
\end{tabular}

is accepted by industry. Moreover, it is more execute efficient, more secure, solves the consensus and is a standardized operating system that have consensus pluggable architecture. Through consensus pluggable architecture, one can use any consensus algorithm.

\section{Reason for Opting Blockchain}

Blockchain conveys solid ongoing data from numerous layers to a large stakeholders, just like the case with tax collection, particularly on a worldwide level. Blockchain is utilized because its distributed ledger which means that Blockchain offers transparency and all supply chain are registered on the system to record all transactions involved in exchange of a product or bulk of products from seller to purchaser. As a result, Blockchain accommodates and maintains all history of transactions performed and nothing can be excluded during performing any transaction. Because of using Blockchain, any organization can improve its renew as the digitized transactions are recorded on untampered ledgers which can never be altered. In case of any alteration, the chain of trust is corrupted that causes the whole network to be compromised. In case of corruption of the network, the peer nodes are not affected as they have their own network which includes ordering nodes. Upon generation of transactions, the ordered nodes computes hashes of the same. The hashes are stored and appended with previous transactions. The final hash generated is disseminated among the other nodes which upon receiving, calculates their own final hash based on their own records. The transactions are accepted or rejected based on the final hash values generated at the nodes. In case of matching of the final hash values, transaction is generally accepted otherwise, the new transaction is rejected.

Focus of any organization functioning in today's competitive marketplace is to gain and sustain competitive advantage. With the huge volumes of data stored in databases, data marts and data warehouses coupled with advanced data analysis tools, managers are now in a better position to make smart and effective decisions which result in competitive advantage for their organizations. Business Analytics (BA) is a new and upcoming area of advanced data analysis that has emerged as a significant area of study for both researchers as well as practitioners over the last two decades. BA is the process of transforming huge volumes of data into new knowledge through analysis and using that knowledge to for effective decision making and problem solving which ultimately results in value-creating competitive actions.

The proposed research work corresponds to the priority areas of Saudi Arabia. According to vision 2030 of Saudi Arabia, the government is investing on Blockchain. Keeping in view the importance and the features it offers, Blockchain is implemented in rest of the world. This research studies contributes in terms of utilizing Blockchain for VAT in Saudi Arabia so that all possible solutions are shifted to Blockchain infrastructure.

The author propose a solution for VAT and supply-chain interface that is built on standardized Hyperledger interface. The proposed VAT model is on execute-order-architecture. Specifically, Hyperledger composer is used to design VAT system and implemented the interface for all the involved parties as shall be explained in the upcoming sections. The

Section II provides background studies conducted regarding VAT collection and various employed techniques for its collection. Section III elaborates methodology of our own proposed solution and provides comprehensive implementation details. Moreover, Blockchain employment in various domains is explained in the section which is followed by details of various configuration and implementation related to employing Blockchain for VATA collection in the Kingdom of Saudi Arabia. Section V concludes the paper.

\section{BACKGROUND}

Along with its partners across the GCC, the Kingdom of Saudi Arabia has chosen to implement a standard VAT tax rate of $5 \%$. This is one of the lowest rates in in the world. See the examples below of standard VAT rates in other countries. Most of the countries chooses to employ some sort tax rate within countries as depicted in Fig. 2.

As can be ascertained from vision 2030 of Saudi Arabia, Government is investing on Blockchain. This research studies contributes in terms of utilizing Blockchain for VAT in Saudi Arabia.

Modern technologies such as artificial intelligence, Internet of Things, Blockchain are among the key pillars of the development and progress of countries and serve as major contributor to the Industry 4.0 and the development of GDP. Encompassed in this vision is Saudi Arabia's ambition to 


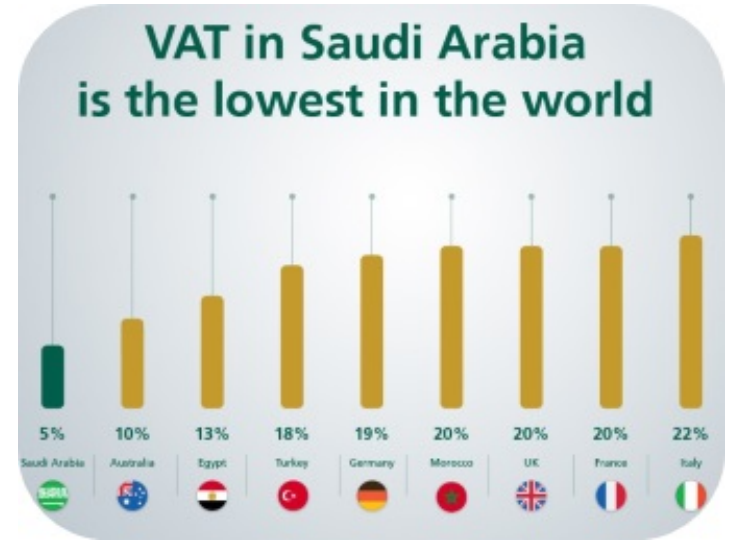

Fig. 2. VAT rates in various countries

deliver an improved e-government, drive digital transformation and attract foreign investment. With a focus to also improve the Kingdom's ease of doing business ranking, achieving these ambitions will require challenges to conventional practices. Implementing modern technologies such as artificial intelligence, Internet of Things, and Blockchain is core to enable this transformation. Furthermore, this path to digital transformation will play a fundamental role in shaping the way challenges such as competition, cost and budget pressures, changing citizen and resident demands, are undertaken. Blockchain, in particular, has the potential to transform government services while disrupting industries such as healthcare, education, banking, and real estate. The technology could reduce VAT Fraud and can reduce VAT Loss [23].

Blockchain offers transparency and all supply chain are registered on the system to record all transactions involved in exchange of a product from seller to purchaser. As a result, Blockchain accommodates and maintains all history of transactions performed and properly records any action/transaction that cannot be excluded or omitted. Because of using Blockchain, any organization can improve its renew as the digitized transactions are recorded on untampered ledgers which can never be altered. In case of any alteration, the chain of trust is corrupted making the whole network compromised. However, in case of corruption of the network, the peer nodes are not affected as they have their own network which includes ordering nodes. The ordering nodes upon generation of transactions compute hashes of the same. The hashes are stored and appended with previously generated transactions. The final hash generated is disseminated among the other nodes which upon receiving calculates their own final hash based on their on records. The transactions are accepted or rejected based on the final hash values generated at the nodes. In case of matching of the final hash values, transaction is generally accepted otherwise, the new transaction is rejected. Peer nodes perform evaluation, validation, they provide a fault tolerant environment in case of a node which is down. Because of the feature under discussion and as provided in the Fig. 3, we feel that the proposed infrastructure can improve the newly adopted VAT manual collection system in terms of generating revenue in Saudi Arabia.

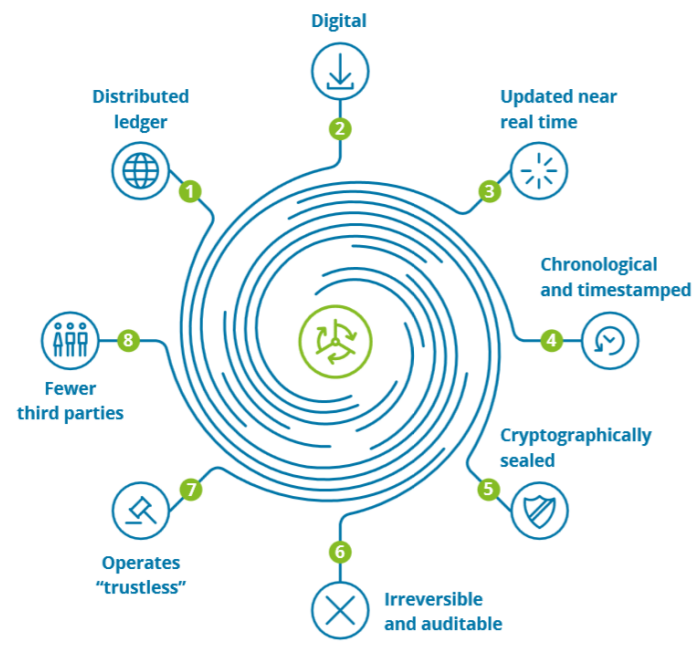

Fig. 3. Blockchain Key Features [24]

\section{A. Analysis and Comparison Results of Employing Various Consensus Algorithms}

The existing solutions for Value Added Tax (VAT) collection are manual and electronic methods. One of these is depicted in Fig. 4.

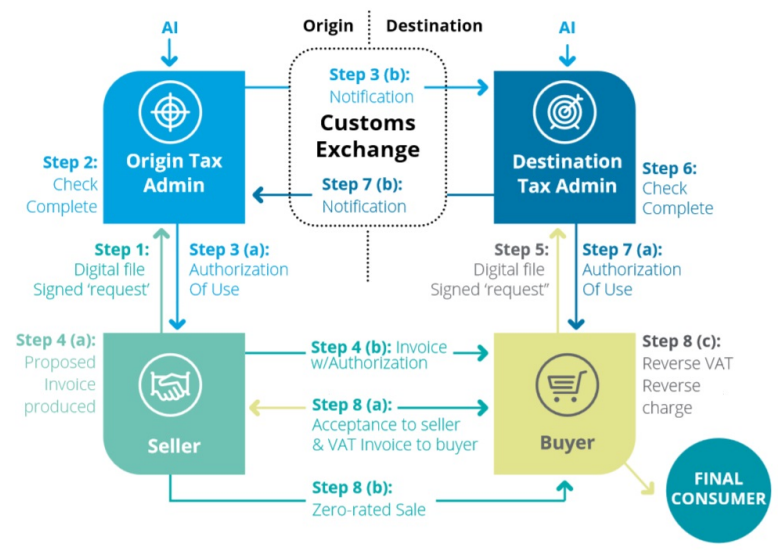

Fig. 4. Electronic Tax Collections [24]

Researchers have provided solutions that are based on Blockchain [11], [25], [26], [22], [27], [28], [29], however, they are using mining as consensus algorithm and are not proven to be very efficient neither they are standardized. Mining based architecture are also known as order-executearchitecture. Wijaya et al. [11] implemented Blockchain through Proof of Work (PoW) as consensus mechanism for implementation and collection of VAT. However, the PoW is not very efficient i.e. transaction throughput is too slow (21 transactions per second).

Wijaya et al. [11] have proposed a convention permissioned based Blockchain that turns around the way toward dealing with fake tax invoices. Their strategy requires Tax Payable for VAT Purposes (TPVP) shall not be allowed to make tax invoices solicitations to different TPVPs without first procuring legitimate tax credit i.e. A TPVP needs to pay some cash to get the tax credits, at that point they can make a duty 
receipt as an approach to exchange the tax credits to another TPVP. By turning around the procedure, it is ensured that the tax invoices in the framework are portrayal of genuine assessment cash flowing through VAT framework. i.e. invoice is issued after checking credit and debit record of VAT through Blockchain. Thus, the paper based mechanism is converted to Blockchain based electronic VAT collection process.

The Blockchain provides an on-line environment for maintaining distributed database of records, ledgers and events that are executed and these belongs to a number of participating bodies. The public ledger and records gets updated and modified with the permission of majority of participants and is not subject to subsequent crash, modification or manipulation. The famous controversial bitcoin is based on Blockchain flawless technology. Blockchain provides a democratic place where it can further facilitate in provision of applications in the domains of finance and others which are briefly explained in the following subsections.

\section{B. The Trust Aspect}

The current business activities are performed through "Trust" on third parties for ensuring transactions are performed flawlessly. However, it is possible that these third parties are hacked, misused or mismanaged. Such issues are better coped through Blockchain wherein at any point in time, the transactions carried out at as the assets are verifiable in future.

\section{Smart Contracts}

Smart contract is another use case of Blockchain which are digital programs that keep conditional records of participants and upon occurring specific event, the contract elements are executed which may include payment to a party.

\section{Smart Property}

Blockchains comes with tremendous opportunities. Use cases of Blockchain also includes "smart property" where the property can be physically or nonphysical. Examples of physical entities may include car, house, plot and things like that. Blockchain has been remained the most secure test environment [30].

\section{E. Health and the Banking Sector}

Most of the banking sectors are exploring ways regarding how to migrate towards Blockchain technology in order to secure their transactional records. Health records, legal documentation, private securities and marriage licenses are maintained in the block-chain effectively. These assets are best protected through digital fingerprints of the assets instead the actual asset [31], [32], [33].

\section{F. Understanding Security Mechanism of Blockchain as Case Study of Bitcoin:}

In Blockchain, security of transactions is ensured through public and private cryptographic keys i.e. transactions are protected through digital signatures. So in order to spend money, the owner of the cryptocurrency needs to prove his ownership of the private key Every transaction is broadcasted to the rest of nodes and then after verification the transaction is recorded in the ledger that is public in nature. In any case, there is question of keeping up the request of these exchanges that are communicated to each other hub in the Bitcoin network. The Bitcoin tackled this issue by an instrument that is currently famously known as the Blockchain framwork. The Bitcoin framework orders exchanges by setting them in blocks and then connecting these in a chain. The exchanges in a single block are considered to have occurred in the meantime. These blocks are connected to one another (like a chain) in an appropriate straight, sequential manner with each block comprising the hash of previous one.

Any node can however contain unconfirmed transactions from which blocks can be created and broadcasted among other nodes. Bitcoin tackles this issue by presenting a scientific riddle: each block will be added in the Blockchain given that it contains a response to an extremely extraordinary numerical query. It takes ten minutes for a node in the Bitcoin system to make a correct speculation and produce a block. A little likelihood exists that more than one blocks are created. To cope this problem, it is checked which node solves the problem that node will be allowed to broadcast the block to all other nodes in the network.

The next section provides necessary details of methodology for the proposed solution of VAT implementation in Saudi Arabia through Blockchain.

\section{METhodology}

The methodology for our proposed solution alongwith selected implementation details are provided in the following subsections:

\section{A. Selection of Blockchain Solution for VAT Collection}

Researchers have provided solutions for businesses on peer-to-peer networks, however, those cannot be adopted as a standard for all. Similarly, they do not have support from industry. Generally, there is always support from industry for the acceptance of a product otherwise failure comes to such products in the long run. The fact can be ascertained by looking to Android mobile operating system which is supported by Google and it has achieved an acceptance and received most of the mobile market share. There were many other operating systems that could not get much popularity due to unavailability of support from industry big players. In this research, the author have opted Blockchain, a solution that can easily be adopted by industry and has quite big support for business applications. After selection of the Blockchain infrastructure, the author designed and implemented VAT system over the Blockchain for Saudi Arabia as a use-case .

\section{B. Proposed Solution and Selected Implementation Details}

The proposed model is primarily designed to implement VAT system for the Kingdom of Saudi Arabia through state-ofthe-Art Blockchain. Ideally, any supply chain management that relates to public and private organizations, service providers, consumers, large and small businesses will essentially be employed through Blockchain. For collection of VAT, the architectural framework is built upon a Hyperledger Fabric operating system, connecting a number of supply chain management organizations. The network comprises of a cluster of 
servers that possess orderer nodes and certificate authorities (CAs), peer nodes. Saudi VAT collection mechanism is hosted on the cluster that manages and control the entire logical components including implementation of mechanism for consensus and certification authority. Likewise a group of interconnected servers implementing supply chain management are connected to the cluster through peer nodes as depicted in Fig. 5.

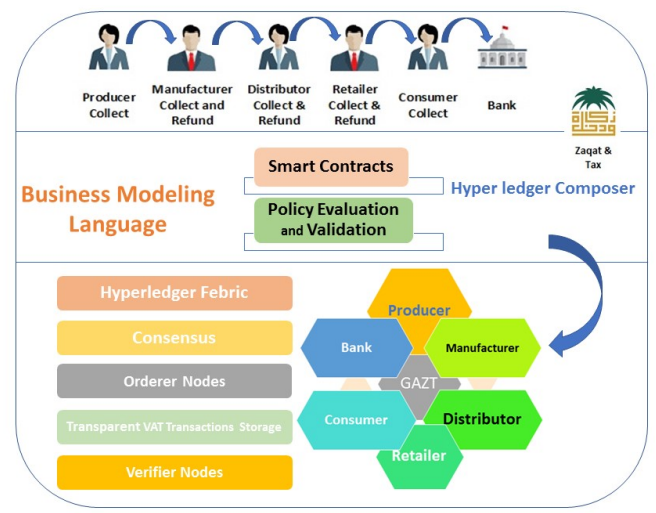

Fig. 5. Complete Proposed VAT System on Blockchain Infrastructure

The Hyperledger Fabric is the main structure or backbone that runs the composer over itself. In the Hyperledger Fabric, a network is composed consisting of cluster of servers for hosting Saudi VAT collection departments and in similar fashion, collection of servers hosting tax payable organizations which are interconnected with each others. The upcoming organization that intends to join the network or become part of the Blockchain are issued certificates by Certificate Authority (CA) of Saudi Arabia for secure communication among peers. These certificates are issued to organizations for a particular role and based on those roles, the transactions are controlled in the Blockchain.

After successful deployment of the Hyperledger Fabric, Hyperledger composer Business Network is deployed on each of the node and likewise issued connection profile for each of the relevant participant in individual organizations. Through these connection profiles, the composer-rest-server API creates URLs for different services including the creation of records or deletion of some records. The organizational personnel can trigger smart-contract transactions to deduct and send VAT amount from their system invoices to the distributed ledger. Inresponse, new automatic transactions are triggered that update VAT amount on-behalf of organization and the payee. In the next section, implementation related details regarding the proposed solution is provided.

\section{IMPLEMENTATION}

In this section, technical details are provided related to VAT implementation initiating with configuration of various services.

\section{A. Configuration of Various Services}

Inside the fabric sample, the directory contents are downloaded and modified to increase number of joining organizations in the Count variable of the file namely, cryptoconfig.yaml. To configure the proposed model application's services on each peer which includes CA, orderer, Peer, couchdb and CLI, the settings are re-configured in the dockercompose.yaml file. In this file, the certification key are added for the organizations that intends to join the network. The author also added extra-hosts section after the volume section in all of our services except Couchdb and CLI. After creation of separate docker-compose.yaml file for each of the other organization. These file will have the configuration of various services for the other organizations. Here for supply chain organization two services will be added, i.e. peern.example.com and couchDB. For deployment on other organization peers the crypto-config folder is copied, docker-composer-peern.yml, env folder and start-peern.sh. To start network on each peer command ./start-peern.sh is utilized.

With a running Hyperledger fabric network and multiple peer nodes, the business network is deployed using Hyperledger composer. To do so first Peer Admin Cards are created for managing the network. In this connection profile, the author added all pool of organizational IPs which are taking part in the network and updated service certificates for them. Further, added these settings in the connection profile in the section of JSON CONNECTION PROFILE SECTION.

To determine whether a particular transaction is valid, Validation System Chaicode (VSCC) is invoked that is accompanied by the transaction's chain code. When a peer receives a transaction, it invokes the VSCC associated with the transaction's Chaincode as part of the transaction validation flow to determine the validity of the transaction.

\section{B. Creation of Participation and Assigning Identities}

In Composer a Participant is just a data item, specifically an object in a Participant Registry. A participant cannot access the Business Network on the Fabric until an Identity has been Issued to and bound to that Participant. Identities are generated by the CA which belongs to an Organization. A user (administrator) with an Identity can create Participants if they have the ACL access to do so, but only an Identity with specific rights in the $\mathrm{CA}$ can issue Identities.

\section{Design of Blockchain based VAT System}

Fig. 6 outlines the Structure of Business Network Definition that runs on composer base architecture. It depicts a complete data model of the proposed architecture that includes Assets, Participants, and Transactions. All stackholders can trigger their transactions, and can explain how data will be managed.

For implementation of the proposed infrastructure, Blockchain environment is built. The state of art tool, the hyperledger fabric is opted to define business logic and configure orderer nodes. Peers have their own stakeholders which includes various supply chains like manufacturer, mediocre.Further, as per our feasibility study, the deployment of such a system is highly effective.

For the purpose, a business-oriented modeling language is opted as the hyperledger fabric interface is so complex for writing applications. The author opted hyperledger composer that is a step further and application oriented. Through this, applications can be modeled more appropriately. The VAT 


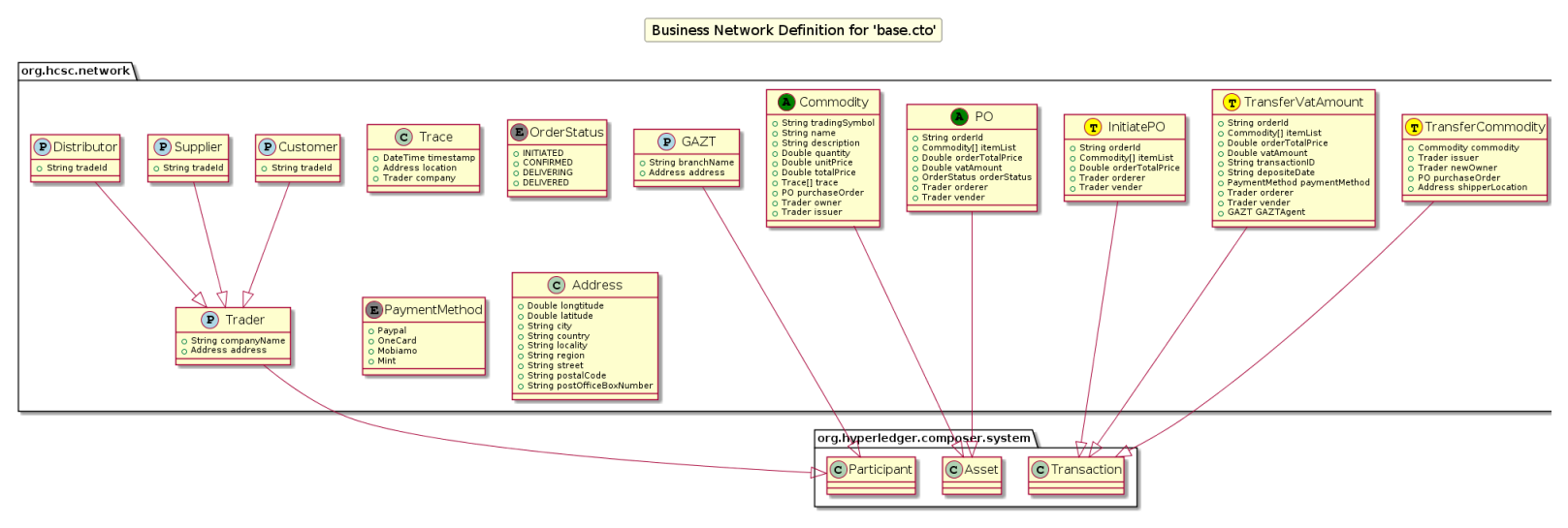

Fig. 6. Class diagram of Business Network Archive

system is designed through hyperledger composer which shall define all components and how they will operate on the modeling language as depicted in Fig. 7.

\section{Deployment of Hyperledger Composer and Peers}

The proposed model is primarily designed to implement Value Added Tax system of the Kingdom of Saudi Arabia through state-of-the-Art Blockchain. Ideally, any supply chain management that relates to public and private organizations, service providers, consumers, large and small businesses and industries will essentially be employed through Blockchain. For collection of VAT, our framework is built upon a Hyperledger Fabric network, which connects all the supply change management organizations in Saudi Arabia. A cluster of servers is framed that possess orderer nodes and certificate authorities (CAs). Saudi VAT collection mechanism is hosted on the cluster that shall essentially be a managing platform to adopt and implement the entire logical and other necessary details of the consensus mechanism and certification authority. Likewise a group of servers as depicted in Fig. 7, which are interconnected with each others implementing supply chain management which are connected to the cluster through peer nodes.

In the Hyperledger Fabric, a network is composed consisting of cluster of servers for hosting Saudi VAT collection departments and collection of servers hosting tax payable organizations which are interconnected with each others. The upcoming organization intended to join the network or become part of the Blockchain are issued certificates by CA of Saudi Arabia. These certificates are issued to organizations for a particular role and based on those roles, the transactions are controlled in the Blockchain.

\section{E. Hyperledger Business Composer Network}

After successful deployment of the Hyperledger Fabric, Hyperledger composer Business Network is deployed on each of the node and likewise connection profiles are issued for each of the relevant participant in individual organizations. Through these connection profiles, the composer-rest-server API creates URLs for different services like creation and deletion of records. Organizational personnel can trigger smartcontract transactions to deduct and send VAT amount from their system invoices to the distributed ledger. In-response new automatic transactions are triggered that update VAT amount on-behalf of organization and the payee.

Inside the fabric sample downloaded from the directory contents are modified to increase number of joining organizations in the Count variable of the file namely, cryptoconfig.yaml. To configure our proposed model application's services on each peer which includes CA, orderer, Peer, couchdb and cli, the settings are re-configured in the dockercompose.yaml file. In this file the certification key are added for the organizations that intends to join the network. Extrahosts section are also added after the volume section in all of our services except Couchdb and CLI. Further, dockercompose.yaml creates separate file for each of the other organization. These file will have the configuration of our services for the other organizations. Here for supply chain organization, only two services are added which are peern.example.com and couchDB. For deployment on other organization peers the folder of crypto-config is copied, docker-composer-peern.yml, env folder and start-peern.sh. To initiate the network on each peer, the command ./start-peern.sh executes the network.

\section{F. Deploying Business Network through Hyperledger Com- poser}

With a running Hyperledger fabric network and multiple peer nodes, the business network is deployed using Hyperledger composer. To do so. first Peer Admin Card is created that manages the network. In this connection profile, authors have added all pool of organization IPs which are taking part in the network and update our services certificates. Further, added these settings in the connection profile in JSON connection profile section.

\section{Conclusion}

Tax-returns and settlements are ascertained over a fixed period, for instance over monthly or quarterly basis and the counts are not based on exchanges, yet rather on self-assertive dates for instance receipt dates. The process is troublesome for governments, if certainly feasible, to track VAT installments. The computerized age is additionally forming tax collection systems into a totally extraordinary shape, by not just changing the connection among citizens and tax authorities, yet in 


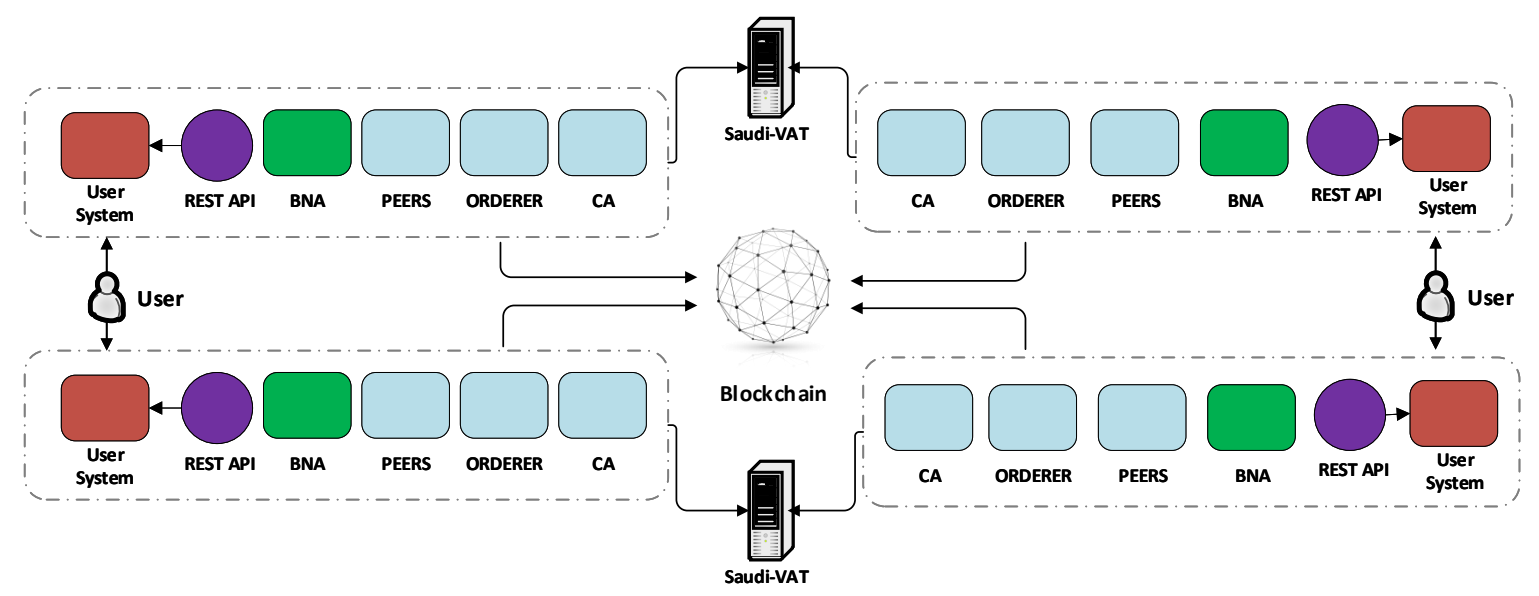

Fig. 7. Proposed Implemented Architecture for Value Added Tax Collection in KSA

addition modifying the manner in which government taxes are covered, submitted and stored. The capability of digitizing VAT has been seen by numerous nations, and new arrangements emerge including SAF-T in Europe or real time invoicing mechanism in South America and Brazil. This search studies provides details of various methods applied for VAT collection through Blockchain and analyses the presented solutions. The studies contributes by presenting a more effective and efficient VAT collection solution through Blockchain. The author, provided a proof-of-concept implementation on Hyperledger composer which is an execute-order architecture to satiate efficient transaction processor over Blockchain.

\section{FUTURE WORK}

Blockchain-based VAT system is a step forward in transparent and secure Tax collection systems. The solution so far presented in the literature are based on Proof-of-Work consensus model that only works with Bitcoin architecture. However, in the proposed architecture VAT is designed and implemented on PBFT (Practical Byzantine Fault Tolerance) consensus model. It is recommended that other consensus model should be enabled on Hyperledger Fabric operating system and run the proposed architecture. That will give some more interesting results on performance, security and storage model of VAT based transactions.

\section{ACKNOWLEDGMENT}

This research is supported by Deanship of Research, Islamic University of Madinah.

\section{REFERENCES}

[1] A. Schenk, V. Thuronyi, and W. Cui, Value added tax. Cambridge University Press, 2015.

[2] J. Olsen, C. Kogler, J. Stark, and E. Kirchler, "Income tax versus value added tax: A mixed-methods comparison of social representations," Journal of Tax Administration, vol. 3, no. 2, pp. 87-107, 2017.

[3] M. Cooper and M. Knittel, "Partial loss refundability: How are corporate tax losses used?" National Tax Journal, pp. 651-663, 2006.

[4] D. Butler et al., "Superannuation: Managing tax losses in an smsf," Taxation in Australia, vol. 49, no. 11, p. 699, 2015.

[5] L. Barbone, M. Bonch-Osmolovskiy, and G. Poniatowski, "Study to quantify and analyse the vat gap in the eu member states," 2015.
[6] K. Pavlov, "Can the general reverse charge mechanism combat missing trader fraud and provide for secure vat collection?" 2017.

[7] F. Times, "Saudi arabia and uae introduce 5[Online]. Available: https://www.ft.com/content/b1742920-efd0-11e7-b220-857e26d1aca4

[8] GAZT, "HealthCare Guideline, version 1, February 2018 by Kingdom of Saudi Arabia," 2018, available at: https: //www.vat.gov.sa/sites/default/files/2018-03/VAT\%20\%20Healthcare\% 20Guide\%20English.pdf.

[9] GAZTAX, "Imports and Exports Guideline, September 18, 1st Edition by Kingdom of Saudi Arabia," 2018, available at: https://www.vat.gov.sa/sites/default/files/2018-09/VAT_import_Export_ Guideline_English.pdf.

[10] I. Bostan, C. Popescu, C. Istrate, I.-B. Robu, and I. Hurjui, "The impact of taxation of the domestic economic transactions on the vat collection through electronic fiscal devices," Amfiteatru Economic, vol. 19, no. 45, p. $581,2017$.

[11] D. A. Wijaya, J. K. Liu, D. A. Suwarsono, and P. Zhang, "A new blockchain-based value added tax system," in International Conference on Provable Security. Springer, 2017, pp. 471-486.

[12] P. Resnick, R. Zeckhauser, J. Swanson, and K. Lockwood, "The value of reputation on ebay: A controlled experiment," Experimental economics, vol. 9, no. 2, pp. 79-101, 2006.

[13] G. Linden, B. Smith, and J. York, "Amazon. com recommendations: Item-to-item collaborative filtering," IEEE Internet computing, no. 1, pp. 76-80, 2003.

[14] M. A. Morid and M. Shajari, "An enhanced e-commerce trust model for community based centralized systems," Electronic Commerce Research, vol. 12, no. 4, pp. 409-427, 2012.

[15] J. Yli-Huumo, D. Ko, S. Choi, S. Park, and K. Smolander, "Where is current research on blockchain technology?-a systematic review," PloS one, vol. 11, no. 10, p. e0163477, 2016.

[16] G. W. Peters and E. Panayi, "Understanding modern banking ledgers through blockchain technologies: Future of transaction processing and smart contracts on the internet of money," in Banking Beyond Banks and Money. Springer, 2016, pp. 239-278.

[17] X. Xu, C. Pautasso, L. Zhu, V. Gramoli, A. Ponomarev, A. B. Tran, and S. Chen, "The blockchain as a software connector," in 2016 13th Working IEEE/IFIP Conference on Software Architecture (WICSA). IEEE, 2016, pp. 182-191.

[18] P. Walker and P. J. Venables, "Cryptographic currency for securities settlement," Jul. 11 2017, uS Patent 9,704,143.

[19] S. Nakamoto, "Bitcoin: A peer-to-peer electronic cash system," 2008.

[20] C. H. Lee and K.-H. Kim, "Implementation of iot system using block chain with authentication and data protection," in 2018 International Conference on Information Networking (ICOIN). IEEE, 2018, pp. 936-940.

[21] A. Baliga, "Understanding blockchain consensus models," Persistent, 2017. 
[22] R. T. Ainsworth and M. Alwohaibi, "Blockchain, bitcoin, and vat in the gcc: The missing trader example," 2017.

[23] V. 20-30, "Saudi Arabia's ambition to deliver an improved egovernment," available at: https://ameinfo.com/money/economy/ saudi-3/.

[24] S. Underwood, "Blockchain beyond bitcoin," Communications of the $A C M$, vol. 59, no. 11, pp. 15-17, 2016.

[25] R. T. Ainsworth and A. Shact, "Blockchain (distributed ledger technology) solves vat fraud," 2016.

[26] M. Swan, Blockchain: Blueprint for a new economy. "O'Reilly Media, Inc.", 2015.

[27] R. T. Ainsworth and M. Alwohaibi, "The first real-time blockchain vatgcc solves mtic fraud," 2017.

[28] P. van der Zwan, "Cryptocurrencies \& vat," Tax Professional, vol. 2018, no. 32, pp. 12-14, 2018.
[29] M. R. Hoffman, "Can blockchains and linked data advance taxation," in Companion of the The Web Conference 2018 on The Web Conference 2018. International World Wide Web Conferences Steering Committee, 2018, pp. 1179-1182.

[30] J. MICHAEL, A. COHN, and J. R. BUTCHER, "Blockchain technology," The Journal, 2018.

[31] X. Yue, H. Wang, D. Jin, M. Li, and W. Jiang, "Healthcare data gateways: found healthcare intelligence on blockchain with novel privacy risk control," Journal of medical systems, vol. 40, no. 10, p. 218, 2016.

[32] M. Mettler, "Blockchain technology in healthcare: The revolution starts here," in e-Health Networking, Applications and Services (Healthcom), 2016 IEEE 18th International Conference on. IEEE, 2016, pp. 1-3.

[33] N. J. Witchey, "Healthcare transaction validation via blockchain proofof-work, systems and methods," Nov. 19 2015, uS Patent App. $14 / 711,740$. 